\title{
Quasiclassical approach to magnetotransport in magnetic inhomogeneous systems
}

\author{
L. Sheng, D. Y. Xing, Z. D. Wang, and Jinming Dong \\ National Laboratory of Solid State Microstructures and Department of Physics, Nanjing University, Nanjing 210093, China \\ and Department of Physics, University of Hong Kong, Hong Kong \\ (Received 12 July 1996; revised manuscript received 1 November 1996)
}

\begin{abstract}
The conventional quasiclassical approach to magnetotransport in magnetic multilayers and magnetic granular solids is found to be suitable only for collinear magnetization configurations. A quantum treatment of electron spin is proposed to improve the quasiclassical theory to be applicable to arbitrary magnetization alignments, in which the electron distribution functions, as well as the electric fields and currents, need to be regarded as spinor matrices which are off-diagonal in spin space of conduction electrons. An extended Boltzmann equation has been used to derive the two-point spinor conductivity in magnetic inhomogeneous systems. The result obtained is found to be identical to that obtained from the real-space Kubo formula, indicating a close link between the reformed quasiclassical approach and the quantum theory. Angular dependence of the current-in-plane magnetoresistance in magnetic superlattices is discussed. [S0163-1829(97)03909-X]
\end{abstract}

\section{INTRODUCTION}

Giant magnetoresistance (MR) in magnetic layered structures and magnetic granular solids has continuously attracted considerable interest for several years. Investigations on this novel phenomenon have led to the question of proper treatment of electronic transport in inhomogeneous systems, where the characteristic lengths of inhomogeneities (thickness of layers, granular size) are comparable to the average mean-free path of the conduction electrons. In these systems transport properties vary from one region to another so that one must find a way of determining the measurable macroscopic quantities from the local ones. For the magnetic inhomogeneous systems the spin-dependent scattering, which is believed responsible for the giant MR effect, gives rise to extra complications in this problem.

Theoretical approaches to magnetic inhomogeneous systems, including quasiclassical ${ }^{1-8}$ and quantum, ${ }^{9-13}$ have been developed to account for the observed giant MR effect. The quasiclassical approach based on the Boltzmann equation was initially applied by Fuchs and Sondheimer ${ }^{14}$ to calculate the resistivity of thin films due to surface roughness. Recently, this method has been extended by Camley and Barnás $^{1}$ to the magnetic layered structures by including the spin-dependent scattering at ferromagnetic (FM)nonmagnetic (NM) interfaces as well as within FM layers. Due to its simplicity, the Camley-Barnás model under an assumption of collinear magnetization configuration (CLMC) has been developed into an analytical description of the magnetotransport for current in the plane (CIP) of the layers in magnetic multilayers. ${ }^{4,5}$ For current perpendicular to the plane (CPP) of the layers, it is found that, spin accumulation and relaxation play an important role in the MR, and for each spin conduction channel the resistivities of the layers are additive and the total conductivity is the sum over the two spin channels. ${ }^{6}$ Very recently, this model has also been applied to magnetic granular systems and reproduced main features of the MR effect. ${ }^{7}$

Most quasiclassical approaches to MR in magnetic multilayers and magnetic granular solids are confined to the con- sideration of the CLMC, i.e., all the FM regions (layers or granules) are assumed to have only two possible magnetization directions, parallel or antiparallel to a fixed spin quantization axis. In the following we wish to show that the quasiclassical approach suitable to CLMC fails in application to other arrangements of magnetic moments. If there is a change in spin quantization axes in a magnetic inhomogeneous system, it is necessary to use a transformation to connect distribution functions of neighbor regions with different quantization axes. Let $g_{\uparrow}^{(1)}$ and $g_{\downarrow}^{(1)}$ denote the distribution functions for electrons with spins parallel and antiparallel to the quantization axis $\mathbf{A}_{1}$, respectively, and $g_{\uparrow}^{(2)}$ and $g_{\downarrow}^{(2)}$ the distribution functions with respect to the quantization axis $\mathbf{A}_{2}$. We have

$$
\left(\begin{array}{c}
g_{\uparrow}^{(2)} \\
g_{\downarrow}^{(2)}
\end{array}\right)=U(\theta)\left(\begin{array}{l}
g_{\uparrow}^{(1)} \\
g_{\downarrow}^{(1)}
\end{array}\right),
$$

where the transformation matrix is given by ${ }^{1}$

$$
U(\theta)=\cos ^{2}\left(\frac{\theta}{2}\right) \hat{1}+\sin ^{2}\left(\frac{\theta}{2}\right) \hat{\sigma}_{x},
$$

with $\hat{1}$ the $2 \times 2$ unit matrix, $\hat{\sigma}_{x}$ the Pauli spin matrix, and $\theta$ the angle between the two spin quantization axes. In Eq. (2), as well as in all subsequent equations, the overhat stands for a $2 \times 2$ matrix in spin space. Consider a system composed of three FM films labeled as $A, B$, and $C$ from left to right, in which films $A$ and $C$ have the same magnetization axis $\mathbf{A}_{\mathbf{1}}$, and film $B$ has the magnetization axis $\mathbf{A}_{\mathbf{2}}$ at an angle of $\theta$ to $\mathbf{A}_{\mathbf{1}}$. If film $B$ is thin enough and both interfaces are perfect (neither reflection nor diffusive scattering), there is no change in the distribution functions as the electrons move from film $A$ to $C$ across $B$, or vice versa. On the other hand, the total transformation of the quantization axes from film $A$ to $C$ can be regarded as a two-step process. The first step is to rotate the quantization axis by an angle of $\theta$, and the next is to rotate it back to its original direction. As a result, the total transformation matrix is given by $U(\theta) U(-\theta)$, which should be equal to a unit matrix. However, it is found 
from Eq. (2) that $U(\theta) U(-\theta)=\left(1-\frac{1}{2} \sin ^{2} \theta\right) \hat{1}+\left(\frac{1}{2} \sin ^{2} \theta\right) \hat{\sigma}_{x}$. This result violates the basic requirement $U(\theta) U(-\theta)=\hat{1}$ except for $\theta=0$ or $\pi$, indicating that the theory is applicable only for the CLMC, i.e., $\theta=0$ or $\pi$. Thus, it is highly desired to improve the present quasiclassical approach to be suitable to noncollinear magnetization configurations (NLMC).

Quantum theory ${ }^{9-13}$ based on the Kubo formula yields a full quantum treatment of the electron spin, and has been applied to inhomogeneous magnetic structures that are oriented noncollinearly. ${ }^{11}$ For arbitrary arrangements of magnetic moments, it is necessary to introduce the currents, electric fields, and conductivity tensors that are off diagonal in spin space of the conduction electrons. Electron spin belongs to pure quantum effect and has no classical analogue. We propose that, for arbitrary magnetization configurations, it is inadequate to view the electron spin as a classical twocomponent vector, as has been done in previous quasiclassical theories. Instead, the electron distribution function should be regarded as a spinor matrix (off diagonal in the spin space). Only in the special case of the CLMC, both the currents and fields can be simultaneously diagonalized so that the spinor distribution function is diagonal in the spin space. Then, it is sufficient to consider the two diagonal elements $g_{\uparrow}$ and $g_{\downarrow}$ associated with the two spin conduction channels.

In this paper we present a quantum treatment of electron spin in the Boltzmann equation approach and improve on it to be applicable to arbitrary magnetization configurations and arbitrary choices of the quantization axis. The reformed quasiclassical theory yields a two-point spinor conductivity which is the same as that derived from the real-space Kubo formula, indicating a close link between the reformed quasiclassical approach and the quantum theory. In the next section, we introduce the reformed quasiclassical model. The two-point spinor conductivity of magnetic inhomogeneous systems is derived in Sec. III. Finally, in Sec. IV we demonstrate the validity of our formalism by discussing the angular dependence of the CIP MR in a magnetic superlattice.

\section{REFORMED QUASICLASSICAL MODEL}

The electron distribution function depends on both position and velocity. Besides, when the spin degree of freedom is included, it should be considered as a $2 \times 2$ matrix in the spin space, whose diagonal and off-diagonal elements are determined by the choice of the quantization axis. We first choose a reference frame of position dependent quantization axes, in which the local quantization axis is always taken along the direction of the local magnetic moment. The spinor distribution function can be written in the form $\hat{f}_{L}(\mathbf{v}, \mathbf{r})=f_{0}(\mathbf{v}) \hat{1}+\hat{g}_{L}(\mathbf{v}, \mathbf{r})$, where $f_{0}$ is the equilibrium distribution and $\hat{g}_{L}$ is the deviation from that equilibrium in the presence of the electric field. The electron transport through an inhomogeneous system is governed by the Boltzmann equation

$$
\mathbf{v} \cdot \nabla \hat{g}_{L}(\mathbf{v}, \mathbf{r})-e \mathbf{v} \cdot \hat{\mathbf{E}}_{L}(\mathbf{r}) \frac{\partial f_{0}}{\partial \varepsilon}=-\left(\frac{\partial \hat{g}_{L}}{\partial t}\right)_{c}
$$

The drift terms on the left hand side of the equation above arises from the spatial inhomogeneity and the electric field, in which $e$ and $\varepsilon=m v^{2} / 2$ are the charge and kinetic energy of the conduction electron, respectively. The effective electric field $\hat{\mathbf{E}}_{L}(\mathbf{r})$, that includes spin accumulation effects, is also a matrix in spin space. The relaxation time approximation can be used to treat the collision term on the right hand side of Eq. (3). In the reference frame of the local quantization axes the relaxation time matrix is diagonal, which is given by

$$
\hat{\tau}(\mathbf{r})=\left(\begin{array}{cc}
\tau_{\uparrow}(\mathbf{r}) & 0 \\
0 & \tau_{\downarrow}(\mathbf{r})
\end{array}\right),
$$

with $\tau_{\uparrow}(\mathbf{r})$ and $\tau_{\downarrow}(\mathbf{r})$ the local relaxation times for spin-up and down electrons, respectively. Taking into account that $\hat{g}_{L}$ and $\hat{\tau}^{-1}$ are not commutative and that all the terms of the Boltzmann equation must be Hermitian, we write the collision term as

$$
\left(\frac{\partial \hat{g}_{L}}{\partial t}\right)_{c}=\frac{1}{2}\left[\hat{\tau}^{-1}(\mathbf{r}), \hat{g}_{L}(\mathbf{v}, \mathbf{r})\right]_{+}
$$

where []$_{+}$stands for an anticommutator. Only under the assumption of the CLMC, the spinor distribution function is diagonal and so $\hat{g}_{L}$ and $\hat{\tau}^{-1}$ become commutative. It then follows that the collision term given in Eq. (5) takes a diagonal form and the diagonal elements are given by $g_{\alpha \alpha}(\mathbf{v}, \mathbf{r}) / \tau_{\alpha}(\mathbf{r})$, where $g_{\alpha \alpha}(\mathbf{v}, \mathbf{r})$ corresponds to the conventional distribution functions for the spin-up and down electrons. In this special case Eq. (3) reduces to the conventional Boltzmann equation. ${ }^{1}$

For arbitrary magnetization alignments, it is inconvenient to use Eq. (3) since the local quantization axis is position dependent. The next step is to make a coordinate transformation from the reference frame of the local quantization axes to a new one with a fixed quantization axis. This amounts to a rotation in three-dimensional space, which can be characterized by the spherical polar angles $[\theta(\mathbf{r}), \varphi(\mathbf{r})]$ subtended by the local quantization axis with respect to the new fixed one. The unitary matrix of such a transformation is given by $^{11}$

$$
\hat{U}(\mathbf{r})=\left(\begin{array}{cc}
\cos (\theta / 2) & \sin (\theta / 2) e^{-i \varphi} \\
-\sin (\theta / 2) e^{i \varphi} & \cos (\theta / 2)
\end{array}\right) .
$$

In the new reference frame, the spinor distribution function and electric field become $\hat{g}(\mathbf{v}, \mathbf{r})=\hat{U}^{\dagger}(\mathbf{r}) \hat{g}_{L}(\mathbf{v}, \mathbf{r}) \hat{U}(\mathbf{r})$, and $\hat{\mathbf{E}}(\mathbf{r})=\hat{U}^{\dagger}(\mathbf{r}) \hat{\mathbf{E}}_{L}(\mathbf{r}) \hat{U}(\mathbf{r})$, respectively. From Eqs. (4) and (6), the spinor inverse relaxation time $\hat{\Delta}(\mathbf{r})=\hat{U}^{\dagger}(\mathbf{r}) \hat{\tau}^{-1}(\mathbf{r}) \hat{U}(\mathbf{r})$ is found to be

$$
\hat{\Delta}(\mathbf{r})=\frac{1}{2}\left[\left(\frac{1}{\tau_{\uparrow}(\mathbf{r})}+\frac{1}{\tau_{\downarrow}(\mathbf{r})}\right)+\left(\frac{1}{\tau_{\uparrow}(\mathbf{r})}-\frac{1}{\tau_{\downarrow}(\mathbf{r})}\right) \hat{\boldsymbol{\sigma}} \cdot \mathbf{m}(\mathbf{r})\right],
$$

where $\hat{\boldsymbol{\sigma}}$ is the Pauli spin vector operator, and $\mathbf{m}(\mathbf{r})$ is the unit vector along the direction of the local magnetization. Substituting Eq. (4) into Eq. (3), and multiplying both sides 
of Eq. (3) by $\hat{U}(\mathbf{r})$ from right and by $\hat{U}^{\dagger}(\mathbf{r})$ from left, we finally obtain the Boltzmann equation in the new reference frame

$$
\mathbf{v} \cdot \nabla \hat{g}(\mathbf{v}, \mathbf{r})+\frac{1}{2}[\hat{\Delta}(\mathbf{r}), \hat{g}(\mathbf{v}, \mathbf{r})]_{+}=e \mathbf{v} \cdot \hat{\mathbf{E}}(\mathbf{r}) \frac{\partial f_{0}}{\partial \varepsilon} .
$$

In order to compare our result with that derived from the quantum theory, ${ }^{11}$ we restrict ourselves to the case where the charge and spin accumulation effects can be completely included in the effective electric field. It is equivalent to an assumption made by Camblong, Levy, and Zhang ${ }^{11}$ that the vertex corrections of the Green's functions can be represented by a local field. Although its applicability to more general cases has yet to be investigated, we can at least safely apply this assumption to the CIP conduction in magnetic multilayers since no spin accumulation effect needs to be taken into account there. Under the assumption of local vertex corrections, $\hat{\mathbf{E}}(\mathbf{r})$ may be determined from the conservation law of the current $\nabla \cdot \hat{\mathbf{j}}(\mathbf{r})=0$ and the boundary condition $\int_{C} \hat{\mathbf{E}}(\mathbf{r}) \cdot d \mathbf{r}=V \hat{1}$, where $V$ is the voltage applied to the outer boundaries of the sample and the line integral is evaluated along the current path $C$ from one boundary to the other. ${ }^{11}$ In the Boltzmann equation approach the spinor current density is given by

$$
\hat{\mathbf{j}}(\mathbf{r})=e m^{3} \int d^{3} v \mathbf{v} \hat{g}(\mathbf{v}, \mathbf{r}),
$$

whose trace $\operatorname{Tr}[\hat{\mathbf{j}}(\mathbf{r})]=\Sigma_{\alpha} \mathbf{j}_{\alpha \alpha}(\mathbf{r})$ corresponds to the measured value.

The form of Eq. (8) is very general. It is invariant in arbitrary choice of the quantization axis and suitable to the NLMC. In the following approach we start from Eq. (8) and, for convenience, adopt the reference frame with a fixed quantization axis that lies along the external magnetic field.

\section{MAGNETIC INHOMOGENEOUS SYSTEMS}

Let us consider a general inhomogeneous magnetic system with infinite size and with arbitrary magnetization alignments. Equation (8) can be solved in a manner similar to that of solving the conventional Boltzmann equation. ${ }^{5}$ In earlier works, ${ }^{1}$ the interface scattering is included phenomenologically through the boundary conditions at the interfaces by using constant transmission, reflection and incoherent scattering coefficients. This oversimplified treatment leads to an underestimation of the resistivity due to interface scattering. ${ }^{10}$ Later investigations ${ }^{2,10}$ show that the problem can be removed by treating the interface scattering more realistically in terms of thin mixing regions. We will employ such a treatment of using the "bulk" scattering within the mixing regions instead of the interfacial scattering at sharp interfaces. For magnetic multilayers, it was pointed out by Hood and Falicov ${ }^{3}$ that the Fermi velocities in different layers are different and there are potential steps at the interfaces, which can cause electron reflection. For ease of comparing below with the quantum theory in the plane-wave approximation, the effect of the potential steps is not taken into account in the present approach. This approximation is considered to be reasonable if the potential steps at the interfaces

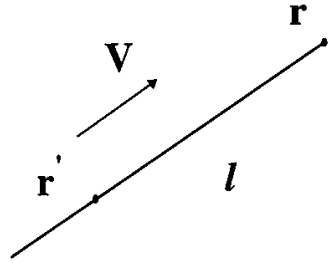

FIG. 1. The integral path $\Gamma\left(\mathbf{r}, \mathbf{r}^{\prime}\right)$ in Eqs. (10)-(12) and (14).

are small enough compared with the Fermi energy so that the reflection at the interfaces can be neglected. It is expected that the present approach can be extended to the case where the potential-step effect needs to be considered.

To solve Eq. (8), we first draw a straight line parallel to $\mathbf{v}$ (designated as $\ell$ ), as shown in Fig. 1. Equation (8) can be regarded as a linear differential equation on line $\ell$, whose solution at point $\mathbf{r}$ is found to be related to that at any other point $\mathbf{r}^{\prime}$ on line $\ell$ through

$$
\begin{aligned}
\hat{g}(\mathbf{v}, \mathbf{r})= & \hat{S}\left(\mathbf{r}, \mathbf{r}^{\prime}\right) \hat{g}\left(\mathbf{v}, \mathbf{r}^{\prime}\right) \hat{S}^{\dagger}\left(\mathbf{r}, \mathbf{r}^{\prime}\right) \\
& +\frac{e}{v} \int_{\Gamma\left(\mathbf{r}, \mathbf{r}^{\prime}\right)} d \ell^{\prime \prime} \hat{S}\left(\mathbf{r}, \mathbf{r}^{\prime \prime}\right) \mathbf{v} \cdot \hat{\mathbf{E}}\left(\mathbf{r}^{\prime \prime}\right) \hat{S}^{\dagger}\left(\mathbf{r}, \mathbf{r}^{\prime \prime}\right) \frac{\partial f_{0}}{\partial \varepsilon},
\end{aligned}
$$

where the spinor propagation factor is given by

$$
\hat{S}\left(\mathbf{r}, \mathbf{r}^{\prime}\right)=P_{\mathbf{r}^{\prime} \rightarrow \mathbf{r}} \exp \left(-\frac{1}{2} \int_{\Gamma\left(\mathbf{r}, \mathbf{r}^{\prime}\right)} d \ell^{\prime \prime} \hat{\xi}\left(\mathbf{r}^{\prime \prime}\right)\right)
$$

Here, $\hat{\xi}(\mathbf{r})=\hat{\Delta}(\mathbf{r}) / v_{F}$ is the inverse mean-free path operator, $\Gamma\left(\mathbf{r}, \mathbf{r}^{\prime}\right)$ stands for the oriented straight path that starts at point $\mathbf{r}^{\prime}$ and ends up at point $\mathbf{r}$, and $P_{\mathbf{r}^{\prime} \rightarrow \mathbf{r}}$ is the pathordering operator along $\Gamma\left(\mathbf{r}, \mathbf{r}^{\prime}\right)$, which reorders the noncommutating $2 \times 2$ scattering matrices in the exponential series from $\mathbf{r}^{\prime}$ to $\mathbf{r}$ and from right to left. ${ }^{11}$ It is easy to see that $\hat{S}\left(\mathbf{r}, \mathbf{r}^{\prime}\right)$ decreases exponentially with the distance $\left|\mathbf{r}-\mathbf{r}^{\prime}\right|$. If taking $\left|\mathbf{r}-\mathbf{r}^{\prime}\right|$ to be large enough in Eq. (10), one finds

$$
\begin{aligned}
\hat{g}(\mathbf{v}, \mathbf{r})= & \lim _{\left|\mathbf{r}^{\prime}-\mathbf{r}\right| \rightarrow \infty}\left(\frac{e}{v} \int_{\Gamma\left(\mathbf{r}, \mathbf{r}^{\prime}\right)} d \ell^{\prime \prime} \hat{S}\left(\mathbf{r}, \mathbf{r}^{\prime \prime}\right) \mathbf{v} \cdot \hat{\mathbf{E}}\left(\mathbf{r}^{\prime \prime}\right)\right. \\
& \left.\times \hat{S}^{\dagger}\left(\mathbf{r}, \mathbf{r}^{\prime \prime}\right) \frac{\partial f_{0}}{\partial \varepsilon}\right)
\end{aligned}
$$

The spinor current at point $\mathbf{r}$ is related to the spinor electric field at point $\mathbf{r}^{\prime}$ by

$$
\mathbf{j}_{\alpha \beta}(\mathbf{r})=\int d^{3} r^{\prime} \vec{\sigma}_{\alpha \beta, \gamma \delta}\left(\mathbf{r}, \mathbf{r}^{\prime}\right) \cdot \mathbf{E}_{\gamma \delta}\left(\mathbf{r}^{\prime}\right),
$$

where $\vec{\sigma}_{\alpha \beta, \gamma \delta}\left(\mathbf{r}, \mathbf{r}^{\prime}\right)$ is a two-point fourth-rank spinor conductivity. In Eq. (13), as well as in all the equations of Sec. IV, summation over repeated Greek indices is implied. To compare Eq. (9), together with Eq. (12), and Eq. (13), it needs to rewrite the line integral in Eq. (12) as an equivalent volume integral by using 


$$
\begin{aligned}
\lim _{\left|\mathbf{r}^{\prime}-\mathbf{r}\right| \rightarrow \infty} \int_{\Gamma\left(\mathbf{r}, \mathbf{r}^{\prime}\right)} d \ell^{\prime \prime} \rightarrow & \frac{2}{\pi} \int d^{3} r^{\prime \prime} \delta\left(\left|\left(\mathbf{r}-\mathbf{r}^{\prime \prime}\right) \times \frac{\mathbf{v}}{v}\right|^{2}\right) \\
& \times \theta\left(\left(\mathbf{r}-\mathbf{r}^{\prime \prime}\right) \cdot \frac{\mathbf{v}}{v}\right),
\end{aligned}
$$

where $\theta(x)$ is the unit step function and $\delta(x)$ is the $\delta$ function. Substituting Eq. (12) together with Eq. (14) into Eq. (9), and comparing it with Eq. (13), we arrive at

$$
\begin{aligned}
\vec{\sigma}_{\alpha \beta, \gamma \delta}\left(\mathbf{r}, \mathbf{r}^{\prime}\right)= & \frac{e^{2} m^{3} v_{F}}{4 \pi^{4}} \int d^{3} v \frac{\mathbf{v} \mathbf{v}}{\left|\mathbf{r}-\mathbf{r}^{\prime}\right|^{2}} \\
& \times \delta\left(|\mathbf{n} \times \mathbf{v}|^{2}\right) \theta(\mathbf{n} \cdot \mathbf{v}) \\
& \times S_{\alpha \gamma}\left(\mathbf{r}, \mathbf{r}^{\prime}\right) S_{\beta \delta}^{*}\left(\mathbf{r}, \mathbf{r}^{\prime}\right) \frac{\partial f_{0}}{\partial \varepsilon},
\end{aligned}
$$

with $\mathbf{n}=\left(\mathbf{r}-\mathbf{r}^{\prime}\right) /\left|\mathbf{r}-\mathbf{r}^{\prime}\right|$ as the unit vector in the direction of $\left(\mathbf{r}-\mathbf{r}^{\prime}\right)$. By performing the integral over the velocity, we finally obtain

$$
\vec{\sigma}_{\alpha \beta, \gamma \delta}\left(\mathbf{r}, \mathbf{r}^{\prime}\right)=\frac{3 C_{D}}{4 \pi} \frac{\mathbf{n n}}{\left|\mathbf{r}-\mathbf{r}^{\prime}\right|^{2}} S_{\alpha \gamma}\left(\mathbf{r}, \mathbf{r}^{\prime}\right) S_{\beta \delta}^{*}\left(\mathbf{r}, \mathbf{r}^{\prime}\right)
$$

where $C_{D}=n e^{2} /\left(2 m v_{F}\right)$ is a constant with dimensions of conductivity/length. Taking Eq. (11) into account, one finds that the present expression (16) for the two-point spinor conductivity is identical to Eq. (3.21) together with Eq. (3.13) of Ref. 11, indicating that the present result obtained from the reformed quasiclassical approach is equivalent to that derived from the Kubo formula in real space.

\section{ANGULAR DEPENDENCE OF CIP MR IN MAGNETIC SUPERLATTICE}

We now consider a magnetic superlattice, whose layers are assumed to lie in the $x-y$ plane and to stack along the $z$ axis. The constitutive relation between field and current, given by Eqs. (13) and (16), simplifies due to the translational invariance in the $x-y$ plane ${ }^{11}$

$$
\mathbf{j}_{\alpha \beta}(z)=\int d z^{\prime} \stackrel{\rightarrow}{\sigma}_{\alpha \beta, \gamma \delta}\left(z, z^{\prime}\right) \cdot \mathbf{E}_{\gamma \delta}\left(z^{\prime}\right)
$$

The reduced one-dimensional conductivity tensor can be obtained as

$$
\vec{\sigma}_{\alpha \beta, \gamma \delta}\left(z, z^{\prime}\right)=\frac{3 C_{D}}{4} \int_{1}^{\infty} d t \overrightarrow{\mathbf{w}}(t) \hat{S}_{\alpha \gamma}\left(z, z^{\prime}\right) \hat{S}_{\beta \delta}^{*}\left(z, z^{\prime}\right),
$$

where

$$
\hat{S}\left(z, z^{\prime}\right)=P_{z^{\prime} \rightarrow z} \exp \left(-\frac{t}{2} \int_{z}^{z^{>}} d z^{\prime \prime} \hat{\xi}\left(z^{\prime \prime}\right)\right)
$$

with $t=v_{F} /\left|v_{z}\right|$ and $z^{<}\left(z^{>}\right)$as the smaller (larger) one of $z$ and $z^{\prime}$, and

$$
\overrightarrow{\mathbf{w}}(t)=\left(\frac{1}{t}-\frac{1}{t^{3}}\right) \mathbf{e}_{\|} \mathbf{e}_{\|}+\frac{2}{t^{3}} \mathbf{e}_{z} \mathbf{e}_{z},
$$

with $\mathbf{e}_{\|}$and $\mathbf{e}_{z}$ the unit vectors in the plane of the layers and in the $z$ direction, respectively.

In what follows we discuss the angular dependence of the CIP MR in magnetic multilayers so as to compare the present quasiclassical theory with the previous one. The dependence of the CIP resistivity on the angle $\theta$ between the magnetizations in successive FM layers has been experimentally investigated in several magnetic layered structures, ${ }^{15-19}$ and it is found that the magnitude of the CIP MR always varies approximately as $\cos ^{2}(\theta / 2)$. On the theoretical side, Vedyayev et ll $^{20}$ have applied the real space Kubo formalism to obtain a linear variation of the CIP MR with $\sin ^{2}(\theta / 2)$ [which is equivalent to a variation proportional to $\left.\cos ^{2}(\theta / 2)\right]$ when there are no potential steps at the interface. Here we wish to show that the present approach can reproduce a linear $\cos ^{2}(\theta / 2)$ dependence of the resistivity $\rho(\theta)$ at least for two important limiting cases, which is in good agreement with experimental data.

We consider a FM/NM superlattice composed of FM layers of thickness $a$ with spin-dependent mean-free path $\lambda_{s}(F)(s=\uparrow, \downarrow)$ and NM layers of thickness $b$ with spinindependent mean-free path $\lambda(N)$. The scattering at the $\mathrm{FM} / \mathrm{NM}$ interfaces is modeled as impurity scattering in thin mixing interlayers of thickness $d(\ll a+b)$ with spindependent mean-free path $\lambda_{s}(I)$. The spin quantization axis is chosen to lie along the external magnetic field direction, so the magnetization direction in the $n$th FM layer can be characterized by the polar angles $\left(\theta / 2, \varphi_{n}\right)$ with $\varphi_{n}=0$ and $\pi$ for $n$ being even and odd numbers, respectively. ${ }^{21}$ In the CIP geometry, the electric field $\hat{\mathbf{E}}(z)$ is a constant and thus the average conductivity of the system is

$$
\sigma(\theta)=\frac{1}{L} \int_{0}^{L} d z \int d z^{\prime} \sigma_{\alpha \alpha, \beta \beta}^{x x}\left(z, z^{\prime}\right),
$$

where $L$ is the thickness of the superlattice system. The MR ratio can be defined as

$$
\frac{\Delta \rho(\theta)}{\Delta \rho_{\max }}=\frac{\rho(\theta)-\rho(\pi)}{\rho(0)-\rho(\pi)}
$$

with $\rho(\theta)=1 / \sigma(\theta)$.

We first discuss the cases where the thickness of the FM films is much larger than the mean-free path, i.e., $a \gg \lambda_{s}(F)$. It can be seen from Eqs. (18) and (19), $\sigma_{\alpha \alpha, \beta \beta}^{x x}\left(z, z^{\prime}\right)$ decreases exponentially with increasing $\left|z-z^{\prime}\right|$, and should vanish if $z$ and $z^{\prime}$ are separated by one FM film or more than one FM films. It then follows from Eq. (21) that the main contribution to the variation of the conductivity $\Delta \sigma(\theta)=\sigma(\theta)-\sigma(\pi)$ comes from the integration with $z$ and $z^{\prime}$ in two adjacent FM layers. Taking this point into account, we find $\Delta \sigma(\theta)$ to be proportional to $\cos ^{2}(\theta / 2)$

$$
\begin{aligned}
\Delta \sigma(\theta)= & A \cos ^{2}\left(\frac{\theta}{2}\right) \int_{1}^{\infty} d t\left(\frac{1}{t^{3}}-\frac{1}{t^{5}}\right) \\
& \times E_{b}\left[\lambda_{\uparrow}(F) T_{\uparrow}-\lambda_{\downarrow}(F) T_{\downarrow}\right]^{2},
\end{aligned}
$$


where $\quad A=3 C_{D} / 2(a+b), \quad E_{b}=\exp [-b t / \lambda(N)], \quad$ and $T_{s}=\exp \left[-d t / \lambda_{s}(I)\right]$ are the momentum-dependent transmission coefficients at the interfaces. Since $\Delta \sigma(\theta)$ given by Eq. (23) contains the factor of $\lambda_{s}(F) /(a+b)$, in the present limit of $\lambda_{s}(F) \ll a$ we obtain

$$
\Delta \rho(\theta) / \Delta \rho_{\max }=\cos ^{2}(\theta / 2) .
$$

We next discuss the homogeneous limit where the superlattice period $(a+b)$ is much shorter than the average electron mean-free path. In this limit the approximate expression for the conductivity can be derived from Eqs. (18)-(21), yielding

$$
\sigma(\theta)=C_{D} \operatorname{Tr}\left(\overline{\hat{\xi}}^{-1}\right)
$$

where $\overline{\hat{\xi}}$ is the average of $\hat{\xi}(z)$. By the use of $\hat{\xi}(z)=\hat{\Delta}(z) / v_{F}$ and Eq. (7), we obtain

$$
\overline{\hat{\xi}}=\frac{1}{2}\left[\left(\frac{1}{\overline{\bar{\lambda}}_{\uparrow}}+\frac{1}{\bar{\lambda}_{\downarrow}}\right)+\left(\frac{1}{\overline{\bar{\lambda}}_{\uparrow}}-\frac{1}{\bar{\lambda}_{\downarrow}}\right) \sigma_{z} \cos \left(\frac{\theta}{2}\right)\right],
$$

where

$$
\frac{1}{\overline{\bar{\lambda}}_{s}}=\frac{1}{a+b}\left(\frac{a}{\lambda_{s}(F)}+\frac{b}{\lambda(N)}+\frac{2 d}{\lambda_{s}(I)}\right)
$$

is the self-averaging mean-free path. By using Eqs. (25) and (26), we arrive at Eq. (24) again.

The present theory has been shown to reproduce the correct linear dependence on $\cos ^{2}(\theta / 2)$ of the CIP MR in the two limiting cases. On the other hand, it is straightforward to show that in the thick FM-film limit the previous quasiclassical approach yields a linear dependence on $\cos ^{2}(\theta / 2)$ of CIP MR ratio as well. However, we find that there is a remarkable deviation from the linear dependence in the homogeneous limit. In Fig. 2 a comparison is made between the result of the present theory (solid line) in the homogeneous limit and that calculated from the previous quasiclassical approach (dashed lines), the latter depending on two dimensionless parameters $N_{s}=\bar{\lambda}_{s} /(a+b)$ with $s=\uparrow$ and $\downarrow$. The

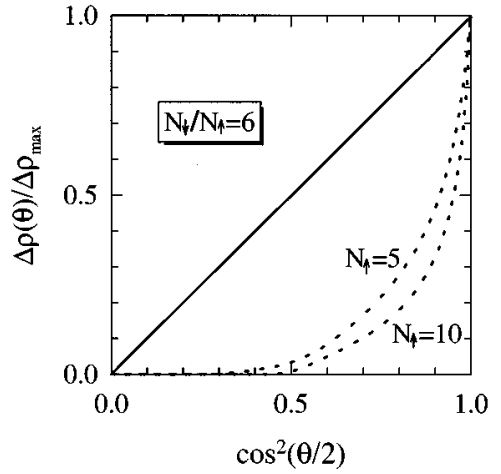

FIG. 2. Angular dependence of the CIP MR ratio for a magnetic superlattice. The solid line represents the linear dependence on $\cos ^{2}(\theta / 2)$ obtained from the present theory, and the dashed lines are results calculated from the previous quasiclassical approach for $N_{\uparrow}=5$ and 10 with fixed spin asymmetric factor $N_{\downarrow} / N_{\uparrow}=\bar{\lambda}_{\downarrow} / \bar{\lambda}_{\uparrow}$.

angular dependence predicted by the previous quasiclassical model is evidently inconsistent with the experimental data of the CIP MR proportional to $\cos ^{2}(\theta / 2)$. In our opinion, this is a glaring example of indicating that the previous quasiclassical approach is inapplicable to NLMC in general cases.

In summary, we have shown that the quasiclassical approach can be reformed to be applicable to arbitrary magnetization arrangements by introducing the spinor distribution function and effective spinor field (off diagonal in spin space) in the Boltzmann equation to describe the electron transport. For a general three-dimensional inhomogeneous system, we have derived the expression for the two-point spinor conductivity and found it to be in agreement with that obtained from the real-space Kubo formula. The present approach is applied to the CIP MR in magnetic superlattices. The calculated result is well consistent with experimental data.

This work was supported by the National Natural Science Foundation and the Doctoral Foundation of National Education Committee in China, and in part by a RGC grant of Hong Kong and a CRCG research grant at the University of Hong Kong.
${ }^{1}$ R. E. Camley and J. Barnás, Phys. Rev. Lett. 63, 664 (1989); J. Barnás, A. Fuss, R. E. Camley, P. Grünberg, and W. Zinn, Phys. Rev. B 42, 8110 (1990).

${ }^{2}$ B. L. Johnson and R. E. Camley, Phys. Rev. B 44, 9997 (1991).

${ }^{3}$ R. Q. Hood and L. M. Falicov, Phys. Rev. B 46, 8287 (1992); R. Q. Hood, L. M. Falicov, and D. R. Penn, ibid. 49, 368 (1994).

${ }^{4}$ M. Liu and D.Y. Xing, Phys. Rev. B 47, 12272 (1993).

${ }^{5}$ L. Sheng and D. Y. Xing, Phys. Rev. B 50, 1001 (1994).

${ }^{6}$ T. Valet and A. Fert, Phys. Rev. B 48, 7099 (1993).

${ }^{7}$ L. Sheng, Z. D. Wang, D. Y. Xing, and J. X. Zhu, Phys. Rev. B 53, 8203 (1996); R. Y. Gu, L. Sheng, D. Y. Xing, Z. D. Wang, and J. M. Dong, ibid. 53, 11685 (1996).

${ }^{8}$ B. Z. Li, J. H. Wu, and F. C. Pu, Acta Phys. Sin. (Oversea Edition) 5, 264 (1996); ibid. 281 (1996).

${ }^{9}$ P. M. Levy, S. Zhang, and A. Fert, Phys. Rev. Lett. 65, 1643
(1990); S. Zhang, P. M. Levy, and A. Fert, Phys. Rev. B 45, 8689 (1992).

${ }^{10}$ H. E. Camblong and P. M. Levy, Phys. Rev. Lett. 69, 2835 (1992).

${ }^{11}$ H. E. Camblong, P. M. Levy, and S. Zhang, Phys. Rev. B 51, 16052 (1995).

${ }^{12}$ P. B. Zhao and F. C. Pu, Phys. Rev. B 51, 11603 (1995).

${ }^{13}$ X. G. Zhang and W. H. Butler, Phys. Rev. B 51, 10085 (1995).

${ }^{14}$ K. Fuchs, Proc. Cambridge Philos. Soc. 34, 100 (1938); H. Sondheimer, Adv. Phys. 1, 1 (1952).

${ }^{15}$ A. Chaiken, G. A. Prinz, and J. J. Krebs, J. Appl. Phys. 67, 4892 (1990).

${ }^{16}$ B. Dieny, V. S. Speriosu, S. S. P. Parkin, B. A. Gurney, D. R. Wilhoit, and D. Mauri, Phys. Rev. B 43, 1297 (1991).

${ }^{17}$ T. Shinjo, in Magnetism and Structure in Systems of Reduced 
Dimension, Vol. 309 of NATO Advanced Study Institute, Series B: Physics, edited by R. F. C. Farrow et al. (Plenum, New York, 1993), p. 323.

${ }^{18}$ M. Patel, T. Fujimoto, E. Gu, C. Daboo, and J. A. C. Bland, J. Appl. Phys. 75, 6528 (1994).

${ }^{19}$ L. B. Steren, A. Barthelemy, J. L. Duvail, A. Fert, R. Morel, F.
Petroff, P. Holody, R. Loloee, and P. A. Schroeder, Phys. Rev. B 51, 292 (1995).

${ }^{20}$ A. Vedyayev, B. Dieny, N. Ryzhanova, J. B. Genin, and C. Cowache, Europhys. Lett. 25, 465 (1994).

${ }^{21}$ K. Wang, S. Zhang, and P. M. Levy, Phys. Rev. B 54, 11965 (1996). 